UCL-IPT-97-11

\title{
Equivalence of the Sine-Gordon and Massive Thirring Models at Finite Temperature
}

\author{
D. Delépine, R. González Felipe and J. Weyers \\ Institut de Physique Théorique \\ Université catholique de Louvain \\ B-1348 Louvain-la-Neuve, Belgium
}

\begin{abstract}
Using the path-integral approach, the quantum massive Thirring and sine-Gordon models are proven to be equivalent at finite temperature. This result is an extension of Coleman's proof of the equivalence between both theories at zero temperature. The usual identifications among the parameters of these models also remain valid at $T \neq 0$.
\end{abstract}




\section{Introduction}

In two dimensional quantum field theories fermionic degrees of freedom can be expressed as bosonic ones and vice versa. A remarkable illustration of this property is the equivalence between the sine-Gordon and (massive) Thirring models [1]. The sine-Gordon model is a $(1+1)$-dimensional field theory of a single scalar field, whose Lagrangian density in Euclidean space-time is defined classically by

$$
\mathcal{L}_{S G}=\frac{1}{2} \partial_{\mu} \varphi \partial_{\mu} \varphi-\frac{\alpha_{0}}{\lambda^{2}}(\cos \lambda \varphi-1)
$$

where $\alpha_{0}$ plays the role of a squared mass, $\lambda$ is a coupling constant and the minimum energy is taken to be zero. On the other hand, the Lagrangian density of the massive Thirring model in Euclidean $(1+1)$-dimensional space-time is given by

$$
\mathcal{L}_{T}=-i \bar{\psi} \not \partial \psi-\frac{1}{2} g^{2}\left(\bar{\psi} \gamma^{\mu} \psi\right)^{2}+i m z \bar{\psi} \psi
$$

where $z$ is a cutoff-dependent constant and the $\gamma_{\mu}$ matrices are taken in the form:

$$
\gamma_{0}=\left(\begin{array}{ll}
0 & 1 \\
1 & 0
\end{array}\right), \gamma_{1}=\left(\begin{array}{cc}
0 & i \\
-i & 0
\end{array}\right), \gamma_{5}=i \gamma_{0} \gamma_{1}
$$

The equivalence between the two models was first derived by Coleman[1] following Klaiber's work on the massless Thirring model[2]: he showed that the perturbation series in the mass parameter $m$ of the Thirring model is termby-term identical with a perturbation series in $\alpha_{0}$ for the sine-Gordon model, provided the following identifications are made [1, 3]:

$$
\begin{gathered}
\frac{4 \pi}{\lambda^{2}}=1+\frac{g^{2}}{\pi}, \\
\bar{\psi} \gamma_{\mu} \psi=i \frac{\lambda}{2 \pi} \epsilon_{\mu \nu} \partial_{\nu} \varphi, \\
i m z \bar{\psi} \psi=-\frac{\alpha_{0}}{\lambda^{2}} \cos (\lambda \varphi) .
\end{gathered}
$$

It is worth noticing that relation (4) expresses a duality symmetry between the Thirring and sine-Gordon models, i.e. a correspondence that relates the strong coupling regime in one theory to the weak coupling one in the other.

Different approaches have been used to prove the equivalence between the above models. Mandelstam[3] rederived Coleman's results by explicitly constructing the operators for creation and annihilation of quantum sine-Gordon solitons. These operators satisfy the anticommutation relations and field equations of the fermionic fields in the massive Thirring model. Alternatively, in the path-integral framework, Coleman's proof can be rederived in a very simple [4] way by making a chiral transformation in the fermionic path-integral variables. 
The results quoted above have been obtained in the usual context of relativistic quantum field theory, namely at zero temperature. An obvious question is whether they remain valid at finite temperature $T$. Although a positive answer might be expected from general arguments (bosonisation at finite $T$ ) contradictory results have been presented in the literature [5]-[ 8$]$.

In this letter we give an explicit proof in the path integral approach [4] of the equivalence between the massive Thirring and sine-Gordon models at finite temperature in precisely the terms of Coleman's original argument at zero temperature. Namely, we show that a perturbative expansion in the mass of the Thirring model is term-by-term identical with a perturbation series in $\alpha_{0}$ of the sine-Gordon model, provided the identifications given in Eqs.(田)-(6) are made.

\section{The massive Thirring and sine-Gordon mod- els at finite temperature}

In this section we shall evaluate the partition functions of the massive Thirring and sine-Gordon models using the imaginary time formalism and the path integral approach at finite temperature 9 .

Let us first consider the massive Thirring model. The Euclidean partition function reads

$$
Z_{T}=N_{0} N_{\beta} \int \mathcal{D} \psi \mathcal{D} \bar{\psi} e^{-\int d^{2} x \mathcal{L}_{T}}
$$

where $\int d^{2} x \equiv \int_{0}^{\beta} d x_{0} \int_{-\infty}^{+\infty} d x_{1}(\beta=1 / T)$ and $\mathcal{L}_{T}$ is given by Eq.(2). $N_{0}$ is an infinite temperature-independent normalization constant, whereas $N_{\beta}$ is a divergent temperature-dependent constant to be determined from the free partition function[9]. The functional integral is performed over fermionic fields satisfying antiperiodic boundary conditions in the time direction,

$$
\psi\left(x_{0}, x_{1}\right)=-\psi\left(x_{0}+\beta, x_{1}\right) .
$$

The first step of the evaluation is to introduce an auxiliary two-component vector field [4]

$$
A_{\mu}=-\frac{1}{g}\left(\epsilon_{\mu \nu} \partial_{\nu} \phi-\partial_{\mu} \eta\right)
$$

where $\phi$ and $\eta$ are two scalar fields satisfying the periodic boundary conditions

$$
\phi\left(x_{0}, x_{1}\right)=\phi\left(x_{0}+\beta, x_{1}\right), \eta\left(x_{0}, x_{1}\right)=\eta\left(x_{0}+\beta, x_{1}\right) .
$$

With the help of these fields, the quartic interaction in $\mathcal{L}_{T}$ can be eliminated,

$$
\exp \left(\frac{g^{2}}{2} \int d^{2} x\left(\bar{\psi} \gamma_{\mu} \psi\right)^{2}\right)=\int \mathcal{D} A_{\mu} \exp \left(-\int d^{2} x\left(\frac{1}{2} A_{\mu}^{2}-g \bar{\psi} / A \psi\right)\right)
$$


and we obtain the effective Lagrangian

$$
\mathcal{L}_{T}=-\bar{\psi}\left[i \not \partial-\gamma_{\mu}\left(\epsilon_{\mu \nu} \partial_{\nu} \phi-\partial_{\mu} \eta\right)\right] \psi+i m z \bar{\psi} \psi+\frac{1}{2 g^{2}}\left[\left(\partial_{\mu} \phi\right)^{2}+\left(\partial_{\mu} \eta\right)^{2}\right] .
$$

We now perform the chiral transformation

$$
\begin{aligned}
& \psi(x)=e^{\gamma_{5} \phi(x)+i \eta(x)} \chi(x), \\
& \bar{\psi}(x)=\bar{\chi}(x) e^{\gamma_{5} \phi(x)-i \eta(x)}
\end{aligned}
$$

with $\chi(x)$ satisfying the boundary condition

$$
\chi\left(x_{0}, x_{1}\right)=-\chi\left(x_{0}+\beta, x_{1}\right)
$$

to obtain

$$
\mathcal{L}_{T}=-\bar{\chi} i \not \partial \chi+i m z \bar{\chi} e^{2 \gamma_{5} \phi(x)} \chi+\frac{1}{2 g^{2}}\left[\left(\partial_{\mu} \phi\right)^{2}+\left(\partial_{\mu} \eta\right)^{2}\right]
$$

To write the partition function in terms of the new variables, the Jacobians of the transformations

$$
\begin{aligned}
\mathcal{D} \psi \mathcal{D} \bar{\psi} & =J_{F} \mathcal{D} \chi \mathcal{D} \bar{\chi} \\
\mathcal{D} A_{\mu} & =J_{A} \mathcal{D} \phi \mathcal{D} \eta
\end{aligned}
$$

have to be properly taken into account. Due to the anomaly and the fact that we perform a chiral transformation, the first Jacobian $J_{F}$ is not trivial. It has been computed at finite temperature in Ref. [10] following Fujikawa's procedure [1] and the result is

$$
J_{F}=e^{-\frac{1}{2 \pi} \int d^{2} x\left(\partial_{\mu} \phi\right)^{2}} .
$$

The bosonic Jacobian $J_{A}$ on the other hand is easily evaluated and one finds

$$
J_{A}=\operatorname{det} \frac{-\nabla^{2}}{g^{2}},
$$

where $\nabla^{2}=\partial_{\mu} \partial_{\mu}$. Note that this bosonic determinant is temperature-dependent and hence its contribution is relevant to the partition function, in contrast to the zero-temperature case where it plays no role and can simply be absorbed in the normalization constant.

Finally we have,

$$
Z_{T}=N_{0} N_{\beta} J_{A} \int \mathcal{D} \chi \mathcal{D} \bar{\chi} \mathcal{D} \phi \mathcal{D} \eta e^{-\int d^{2} x \mathcal{L}_{T}}
$$

with

$$
\mathcal{L}_{T}=-\bar{\chi} i \not \partial \chi+i m z \bar{\chi} e^{2 \gamma_{5} \phi(x)} \chi+\frac{1}{2 \kappa^{2}}\left(\partial_{\mu} \phi\right)^{2}+\frac{1}{2 g^{2}}\left(\partial_{\mu} \eta\right)^{2}
$$


and

$$
\kappa^{2}=\frac{g^{2}}{1+g^{2} / \pi}
$$

The integration is performed over fields satisfying the boundary conditions (10) and (13). As can be seen, the $\eta$ field decouples completely and thus can be trivially integrated out.

In order to show the equivalence between the massive Thirring and the sineGordon models, let us expand $Z_{T}$ in the mass parameter $z m$,

$$
\begin{aligned}
Z_{T}= & N_{0} N_{\beta} J_{A}^{1 / 2} \int \mathcal{D} \chi \mathcal{D} \bar{\chi} \mathcal{D} \phi e^{-\int d^{2} x\left(-\bar{\chi} i \not \chi \chi+\frac{1}{2 \kappa^{2}}\left(\partial_{\mu} \phi\right)^{2}\right)} \\
& \sum_{n=0}^{\infty} \frac{(-i z m)^{n}}{n !} \prod_{j=1}^{n} \int d^{2} x_{j} \bar{\chi}\left(x_{j}\right) e^{2 \gamma_{5} \phi\left(x_{j}\right)} \chi\left(x_{j}\right) .
\end{aligned}
$$

Or, equivalently,

$$
Z_{T}=Z_{F D} \operatorname{det}\left(\frac{\kappa}{g}\right) \sum_{n=0}^{\infty} \frac{(-i z m)^{n}}{n !}\left\langle\prod_{j=1}^{n} \int d^{2} x_{j} \bar{\chi}\left(x_{j}\right) e^{2 \gamma_{5} \phi\left(x_{j}\right)} \chi\left(x_{j}\right)\right\rangle
$$

where \langle\rangle denotes the thermal average over the unperturbed ensemble and $Z_{F D}$ is the Fermi-Dirac distribution for massless fermions [9]:

$$
\ln Z_{F D}=2 \int_{0}^{\infty} \frac{d k}{2 \pi}\left\{\frac{\beta k}{2}+\ln \left(1+e^{-\beta k}\right)\right\} .
$$

At this stage, it is worth noticing that Eq. (22) reproduces in the limit $m=0$ the partition function for the massless Thirring model at finite temperature [12, 13].

To evaluate the thermal averages in Eq.(22) we need the boson and fermion free propagators at finite temperature. In the imaginary time formalism and using the Schwinger representation [14], the propagator for a scalar with mass $\mu$ can be defined as

$$
D(x-y)=\frac{1}{\beta} \sum_{n=-\infty}^{+\infty} \int \frac{d k_{1}}{2 \pi} e^{-i k(x-y)} \int_{0}^{\infty} d s e^{-s\left(k^{2}+\mu^{2}\right)},
$$

with $k^{2}=k_{0}^{2}+k_{1}^{2}$ and $k_{0}=2 \pi n / \beta$ - the Matsubara frequencies. Eq.(24) can be expressed in terms of the modified Bessel function $K_{0}(z)$,

$$
D(x-y)=\frac{1}{2 \pi} \sum_{n=-\infty}^{+\infty} K_{0}\left(\mu \sqrt{\left(x_{0}-y_{0}-n \beta\right)^{2}+\left(x_{1}-y_{1}\right)^{2}}\right) .
$$


When $\mu \rightarrow 0$, we get from the last equation

$$
\begin{aligned}
D(x-y) & =-\frac{1}{2 \pi} \sum_{n=-\infty}^{+\infty} \ln \left(\mu \sqrt{\left(x_{0}-y_{0}-n \beta\right)^{2}+\left(x_{1}-y_{1}\right)^{2}}\right) \\
& =-\frac{1}{2 \pi} \ln \left(\mu \beta \sqrt{\cosh \left(\frac{2 x_{1} \pi}{\beta}\right)-\cos \left(\frac{2 x_{0} \pi}{\beta}\right)}\right)
\end{aligned}
$$

which, as required, is periodic in $x_{0}$. Note that the small mass $\mu$ has been added to avoid infrared divergences.

Similarly, the fermionic propagator can be defined at finite temperature as

$$
\begin{aligned}
S(x-y) & =\frac{1}{\beta} \sum_{n=-\infty}^{+\infty} \int \frac{d k_{1}}{2 \pi} e^{-i k(x-y)}\left(k_{0} \gamma_{0}+k_{1} \gamma_{1}\right) \int_{0}^{\infty} d s e^{-s k^{2}} \\
& \equiv i \gamma_{0} S_{0}(x-y)+i \gamma_{1} S_{1}(x-y)
\end{aligned}
$$

with $k_{0}=(2 n+1) \pi / \beta$. The functions $S_{0}(x)$ and $S_{1}(x)$ can be expressed as:

$$
\begin{aligned}
& S_{0}(x)=-\frac{1}{\beta} \frac{\cosh \left(\frac{x_{1} \pi}{\beta}\right) \sin \left(\frac{x_{0} \pi}{\beta}\right)}{\cosh \left(\frac{2 x_{1} \pi}{\beta}\right)-\cos \left(\frac{2 x_{0} \pi}{\beta}\right)}, \\
& S_{1}(x)=-\frac{1}{\beta} \frac{\sinh \left(\frac{x_{1} \pi}{\beta}\right) \cos \left(\frac{x_{0} \pi}{\beta}\right)}{\cosh \left(\frac{2 x_{1} \pi}{\beta}\right)-\cos \left(\frac{2 x_{0} \pi}{\beta}\right)} .
\end{aligned}
$$

$S(x-y)$ is thus antiperiodic in $x_{0}$ with a period equal to $\beta$, as it should be.

The scalar and fermion propagators can be rewritten in a more familiar way, using the following dimensionless "generalized coordinates" $Q \equiv\left(Q_{0}, Q_{1}\right)$,

$$
\begin{aligned}
& Q_{0}(x)=-\cosh \left(\frac{x_{1} \pi}{\beta}\right) \sin \left(\frac{x_{0} \pi}{\beta}\right), \\
& Q_{1}(x)=-\sinh \left(\frac{x_{1} \pi}{\beta}\right) \cos \left(\frac{x_{0} \pi}{\beta}\right) .
\end{aligned}
$$

and

$$
Q^{2}(x) \equiv Q_{0}^{2}(x)+Q_{1}^{2}(x)=\cosh \left(\frac{2 x_{1} \pi}{\beta}\right)-\cos \left(\frac{2 x_{0} \pi}{\beta}\right) .
$$

In terms of these generalized coordinates, $D(x)$ and $S(x)$ are now given by

$$
\begin{gathered}
D(x)=-\frac{1}{2 \pi} \ln (\mu \beta|Q(x)|), \\
S(x)=\frac{i}{\beta} \frac{Q(x)}{Q^{2}(x)} .
\end{gathered}
$$

Introducing the composite operators

$$
\sigma^{+}=\bar{\chi} \frac{1+\gamma_{5}}{2} \chi, \sigma^{-}=\bar{\chi} \frac{1-\gamma_{5}}{2} \chi
$$


and using the relation

$$
\bar{\chi} e^{2 \gamma_{5} \phi} \chi=e^{2 \phi} \sigma^{+}+e^{-2 \phi} \sigma^{-}
$$

Eq.(22) now reads

$$
\begin{aligned}
Z_{T}= & Z_{F D} \operatorname{det}\left(\frac{\kappa}{g}\right) \sum_{n=0}^{\infty} \frac{(-i z m)^{2 n}}{n !^{2}} \prod_{j=1}^{n} \int d^{2} x_{j} d^{2} y_{j} \\
& \left\langle e^{-2\left(\phi\left(x_{j}\right)-\phi\left(y_{j}\right)\right)} \sigma^{+}\left(x_{j}\right) \sigma^{-}\left(y_{j}\right)\right\rangle .
\end{aligned}
$$

To compute the bosonic thermal average, we use Wick's theorem and the well-known identity

$$
\mathcal{T}\left(e^{-i \int d^{2} x j(x) \phi(x)}\right)=: e^{-i \int d^{2} x j(x) \phi(x)}: e^{-\frac{1}{2} \int d^{2} x d^{2} y j(x) \Delta(x-y) j(y)},
$$

where $\mathcal{T}$ is the $x_{0}$-ordering chronological product and : : denotes the normal product; $\Delta(x-y)$ is the propagator of the $\phi$ field and $j(x)$, any space-time function. We have then

$$
\left\langle\prod_{j=1}^{n} e^{-2\left(\phi\left(x_{j}\right)-\phi\left(y_{j}\right)\right)}\right\rangle_{\text {renorm. }}=\frac{\prod_{i>j}^{n}\left(\rho^{2} \beta^{2}\left|Q\left(x_{i}-x_{j}\right)\right|\left|Q\left(y_{i}-y_{j}\right)\right|\right)^{-\frac{2 \kappa^{2}}{\pi}}}{\prod_{i, j}^{n}\left(\rho \beta\left|Q\left(x_{i}-y_{j}\right)\right|\right)^{-\frac{2 \kappa^{2}}{\pi}}}
$$

where $\rho$ is a renormalization scale, $Q\left(x_{i}\right)$ are the generalized coordinates defined in Eqs. (30) and $\kappa$ is given by Eq.(20). The presence of the latter factor is due to the kinetic term of the scalar Lagrangian (cf. Eq.(19)).

The fermionic average is also easily evaluated, if we recall the identity 15]

$$
(-1)^{n+1} \operatorname{det} \frac{1}{f\left(w_{i}-w_{j}^{\prime}\right)}=\frac{\prod_{i<j} f\left(w_{i}-w_{j}\right) f\left(w_{i}^{\prime}-w_{j}^{\prime}\right)}{\prod_{i, j} f\left(w_{i}-w_{j}^{\prime}\right)},
$$

which holds only for the analytic functions $f(w)=w$ and $f(w)=\sinh (\alpha w)$. In our case

$$
Q_{0}=\frac{i}{2}(\sinh w-\sinh \bar{w}), Q_{1}=-\frac{1}{2}(\sinh w+\sinh \bar{w}), Q^{2}=\sinh w \sinh \bar{w}
$$

with $w=\left(x_{1}+i x_{0}\right) \pi / \beta$, and thus

$$
\left\langle\prod_{j=1}^{n} \sigma^{+}\left(x_{j}\right) \sigma^{-}\left(y_{j}\right)\right\rangle=(-1)^{n} \frac{\prod_{i>j}^{n}\left(\beta^{2}\left|Q\left(x_{i}-x_{j}\right)\right|\left|Q\left(y_{i}-y_{j}\right)\right|\right)^{2}}{\prod_{i, j}^{n}\left(\beta\left|Q\left(x_{i}-y_{j}\right)\right|\right)^{2}} .
$$


Substituting Eqs.(38) and (41) into $Z_{T}$, we obtain finally

$$
\begin{aligned}
Z_{T}= & Z_{F D} \operatorname{det}\left(\frac{\kappa}{g}\right) \sum_{n=0}^{\infty} \frac{(z m)^{2 n}}{n !^{2}}\left(\prod_{j=1}^{n} \int d^{2} x_{j} d^{2} y_{j}\right) \\
& \frac{\prod_{i>j}^{n}\left(\rho^{2} \beta^{2}\left|Q\left(x_{i}-x_{j}\right)\right|\left|Q\left(y_{i}-y_{j}\right)\right|\right)^{2-\frac{2 \kappa^{2}}{\pi}}}{\prod_{i, j}^{n}\left(\rho \beta\left|Q\left(x_{i}-y_{j}\right)\right|\right)^{2-\frac{2 \kappa^{2}}{\pi}}} .
\end{aligned}
$$

To compare $Z_{T}$ with the sine-Gordon model, we have to expand in $\alpha_{0}$ the sine-Gordon partition function

$$
Z_{S G}=N_{0} N_{\beta}^{\prime} \int \mathcal{D} \varphi e^{-\int d^{2} x \mathcal{L}_{S G}},
$$

where $\mathcal{L}_{S G}$ is given in Eq.(何) and the integration runs over scalar fields periodic in the time direction:

$$
\varphi\left(x_{0}, x_{1}\right)=\varphi\left(x_{0}+\beta, x_{1}\right) .
$$

This perturbative expansion in $\alpha_{0}$ yields

$$
Z_{S G}=Z_{B E} \sum_{n=0}^{\infty} \frac{1}{n !^{2}}\left(\frac{\alpha_{0}}{\lambda^{2}}\right)^{2 n}\left\langle\prod_{j=1}^{n} \int e^{i \lambda \varphi\left(x_{j}\right)} e^{-i \lambda \varphi\left(y_{j}\right)} d^{2} x_{j} d^{2} y_{j}\right\rangle,
$$

where

$$
\ln Z_{B E}=-\int_{0}^{\infty} \frac{d k}{2 \pi}\left\{\frac{\beta k}{2}+\ln \left(1-e^{-\beta k}\right)\right\}
$$

is the Bose-Einstein distribution for massless bosons. Comparing Eqs.(23) and (46) it is straightforward to check that $Z_{B E}=C Z_{F D}$, where $C$ is an irrelevant (infinite) constant related to the zero-point energies.

Evaluating the bosonic thermal average with the help of relation (37), we obtain

$$
\begin{aligned}
Z_{S G}= & Z_{B E} \sum_{n=0}^{\infty} \frac{1}{n !^{2}}\left(\frac{\zeta \alpha_{0}}{\lambda^{2}}\right)^{2 n}\left(\prod_{j=1}^{n} \int d^{2} x_{j} d^{2} y_{j}\right) \\
& \frac{\prod_{i>j}^{n}\left(\beta^{2} M^{2}\left|Q\left(x_{i}-x_{j}\right)\right|\left|Q\left(y_{i}-y_{j}\right)\right|\right)^{\frac{\lambda^{2}}{2 \pi}}}{\prod_{i, j}^{n}\left(M \beta\left|Q\left(x_{i}-y_{j}\right)\right|\right)^{\frac{\lambda^{2}}{2 \pi}}}
\end{aligned}
$$

with $M$ an arbitrary scale and $\zeta$ is an ultraviolet-cutoff-dependent coefficient.

Comparing $Z_{T}$ and $Z_{S G}$, we see that the two partition functions are identical provided the relations

$$
\begin{aligned}
\frac{4 \pi}{\lambda^{2}} & =1+\frac{g^{2}}{\pi}, \\
\frac{\zeta \alpha_{0}}{\lambda^{2}} & =z m, \\
M & =\rho
\end{aligned}
$$


are satisfied. The first relation is independent of the renormalization scheme, while the last two equations depend on it and hence have only a conventiondependent meaning. We also recover Coleman's relations (5) and (6) between the two theories.

In conclusion, we have shown using the path integral method that the compactification of the time variable into a circle of radius $\beta=1 / T$ preserves the equivalence between the sine-Gordon and massive Thirring models in Coleman's sense: at fixed radius $\beta$ (or at fixed temperature), the perturbation series in the mass parameter of the Thirring model is term-by-term identical with a perturbation series in $\alpha_{0}$ for the sine-Gordon model, provided the identifications given in Eqs.(4)-(6) are made.

\section{References}

[1] S. Coleman, Phys. Rev. D 11 (1975) 2088.

[2] B. Klaiber, in Lectures in Theoretical Physics, Boulder, 1967, ed. by A. Barut and W. Brittin (Gordon and Breach, New York, 1968).

[3] S. Mandelstam, Phys. Rev. D 11 (1975) 3026.

[4] C.M. Naón, Phys. Rev. D 31 (1985) 2035.

[5] D. Wolf, J. Zittartz, Z. Phys. B 51 (1983) 65.

[6] T.R. Klassen and E. Melzer, Int. J. Mod. Phys. A 8 (1993) 4131.

[7] H. Itoyama and P. Moxhay, Phys. Rev. Lett. 65 (1990) 2102.

[8] T. Fujita and H. Takahashi, Finite size corrections in massive Thirring model, preprint hep-th/9706061.

[9] C.W. Bernard, Phys. Rev. D 9 (1974) 3312.

[10] M. Reuter and W. Dittrich, Phys. Rev. D 32 (1985) 513.

[11] K. Fujikawa, Phys. Rev. Lett. 42 (1979) 1195; Phys. Rev. D 21 (1980) 2848.

[12] F. Ruiz Ruiz and R.F. Alvarez-Estrada, Phys. Rev. D 35 (1987) 3161.

[13] M.V. Manías, C.M. Naón and M.L. Trobo, Path-integral fermion-boson decoupling at finite temperature, preprint hep-th/9701160.

[14] J. Schwinger, Phys. Rev. 82 (1951) 664.

[15] J. Zinn-Justin, Quantum Field Theory and Critical Phenomena (Clarendon Press, Oxford, 1996). 